\title{
Theoretical research of friction factor in hydraulically smooth pipes
}

\author{
Mykola Khlapuk $^{1}$, Olexander Bezusyak ${ }^{1}$, Liubov Volk ${ }^{*}$, and Zelu Zhang ${ }^{2}$ \\ ${ }^{1}$ National University of Water and Environmental Engineering, Department of Hydraulic Engineering and Hydraulics, Rivne, 33028 , \\ Ukraine \\ ${ }^{2}$ Inner Mongolia Agricultural University, No. 306, Zhaowuda Road, Saihan District, Hohhot, Inner Mongolia, 010018, China
}

\begin{abstract}
The paper presents the disclosure of the problem of calculating the friction factor. This problem exists in the calculations of head losses for a given flow discharge and the geometric parameters of the pipes. The analysis of the formulas recommended by known scientists is described. The article also presents the shortcomings of the formulas and the variance of the adequacy of the experimental data. These research data were obtained by J. Nikuradze for smooth pipes. We obtained a formula based on the method of dimensional analysis. This formula characterizes the inner surface of the pipes. Also, this formula describes the change in the friction factor from the Reynolds number. The accuracy of calculating the obtained formula is higher than the accuracy of existing formulas.
\end{abstract}

\section{Introduction}

To solve hydrodynamic problems, it is necessary to improve the hydraulic calculation of pipes. Due to the difference in geodetic marks or due to the energy that is transferred to the fluid when passing through the pumps, the fluid moves in the pipes. Hydraulic calculation of pipes is necessary to determine the head loss for the geometric dimensions of the pipes and a given of the flow discharge.

In 1752, Leonard Euler mathematically derived the Bernoulli equation for an elementary stream of real fluid

$$
z_{1}+\frac{p_{1}}{\rho g}+\frac{u_{1}^{2}}{2 g}=z_{2}+\frac{p_{2}}{\rho g}+\frac{u_{2}^{2}}{2 g}+h_{w},
$$

where, respectively, in sections 1 and $2 z_{1}$ and $z_{2}$ - the position of the elementary stream relative to the plane of comparison, $\mathrm{m} ; p_{1}$ and $p_{2}$ - the value of pressure, $\mathrm{Pa} ; u_{1}$ and $u_{2}$ - flow velocity, $\mathrm{m} / \mathrm{s} ; \rho-$ density of liquid, $\mathrm{kg} / \mathrm{m}^{3}$; $g$ - density of liquid, $g=9,81 \mathrm{~kg} / \mathrm{m}^{3}$; - acceleration of gravity, $\mathrm{m} / \mathrm{s}^{2}$. The equation includes the head loss $h_{w}$ that occurs when the fluid moves due to friction forces.

In $1845 \mathrm{~J}$. Weisbach and in $1857 \mathrm{~A}$. Darcy proposed a formula for calculating the head loss [1]

$$
h_{w}=\lambda \frac{l}{d} \frac{\bar{u}^{2}}{2 g}
$$

where $\lambda$ - friction factor; $\bar{u}$ - average flow velocity, $\mathrm{m} / \mathrm{s}$, $\bar{u}^{2} /(2 g)$ - velocity head, $\mathrm{m}$.

Different types of flow are different forms of movement of flow particles. Flow types were experimentally studied by $\mathrm{O}$. Reynolds. He identified the existence of two forms of flow. At low flow velocities, a layered flow is observed, and at high velocities in the form of small vortices with stirring. The first form of flow is called laminar flow, and the second form of flow is turbulent flow.

Classical experiments of O. Reynolds in 1868 and other scientists have shown that with a gradual increase in velocity, the laminar flow is maintained up to a certain flow velocity. After this velocity, the flow turned into a turbulent flow. When conducting experiments in reverse order, ie with decreasing velocity, the turbulent flow was also maintained up to a certain velocity, after which it passed into a laminar flow. O. Reynolds called this velocity critical, at which there is a change in flow. There are two critical velocities: the lower velocity $\bar{u}_{l . v}$, at which the turbulent flow turns into laminar, and the upper velocity $\bar{u}_{u . v}$, at which the laminar flow turns into turbulent. There is always inequality $\bar{u}_{l . v}<\bar{u}_{u . v}$.

For different types of flow, there are different dependencies between head losses $h_{w}$ and average velocity $\bar{u}$. Figure 1 shows a graph of dependence $i=$ $h_{w} / l=f(\bar{u})$ in logarithmic coordinates.

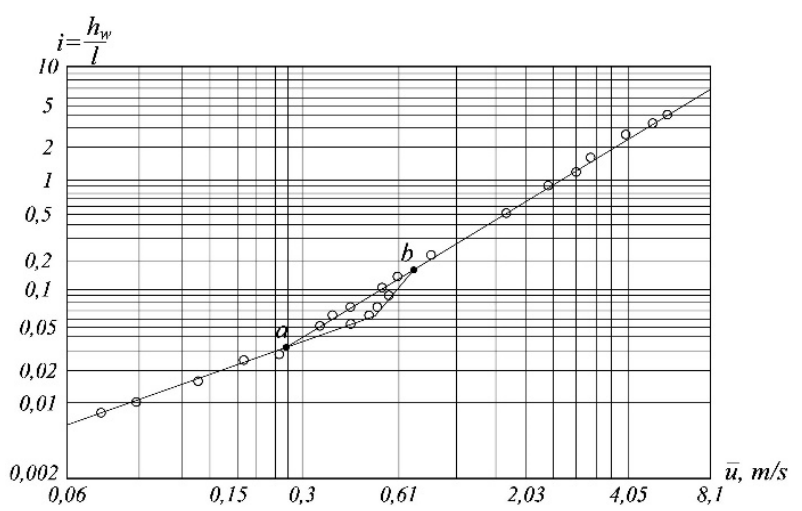

Fig. 1. Graphs of dependence $i=h_{w} / l=f(\bar{u})$.

* Corresponding author: 1.r.volk@ukr.net 
In laminar flow, the graph values of velocity less than $\bar{u}_{l . v}$ (point a) pressure loss along the length proportional to the first degree of velocity, and in turbulent, velocity values greater than $\bar{u}_{u . v}$ (point b) - flow velocities proportional to degree $m$, ranging from 1,75 to 2 . Between points $a$ and $b$ there is a transition zone within which there is a transition of a laminar stream to turbulent, or on the contrary.

Subsequent studies have shown that the presence of a laminar or turbulent flow depends not only on the flow velocity but also on the flow viscosity and on the geometric dimensions of the flow cross section. Therefore, the type of flow is characterized by the value of the dimensionless similarity criterion, which is called the Reynolds number

$$
R e=\frac{\bar{u} d}{v},
$$

where $d$ - pipe diameter, $\mathrm{m} ; \boldsymbol{v}$ - kinematic viscosity of the flow, $\mathrm{m}^{2} / \mathrm{s}$.

In practice, the type of flow in the pressure pipes is determined by the lower critical Reynolds number $\left(R e_{H . \kappa}=\bar{u}_{H . \kappa} d / v=2320\right)$.

Extensive experimental studies of the dependence of the friction factor on the Reynolds number and the smoothness of the inner surface of the pipes in 1928-29 were performed by J. Nikuradze at the Kaiser-Wilhelm Institute under the direction of Dr. L. Prandtl [2,3].

J. Nikuradze investigated the influence of the Reynolds number on the friction factor in pipes with uniform granular roughness and in hydraulically smooth pipes on hydraulic installations [2, 3]. The results of his study of pipes with a uniform granular roughness are shown in Figure 2 in the form of graphs in coordinates $\lg (100 \lambda)=f\left(\lg R e, r_{0} / \varepsilon\right)$.

The importance of this figure 2 , which is called the Nikuradze graph, is that it shows the boundaries of laminar and turbulent flows and regions of hydraulically smooth, prequadratic and quadratic turbulence and the change in friction factor for each region.

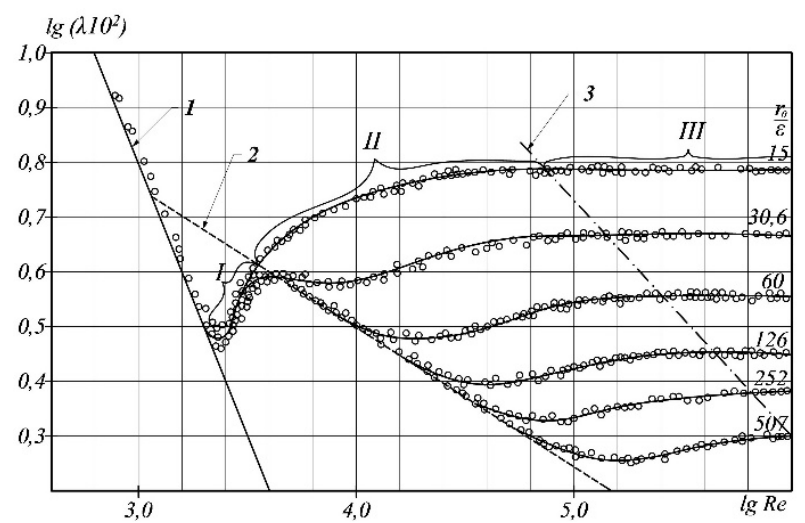

Fig. 2. Graphs of the dependence of the friction factor on the Reynolds number and the inner surface of the pipes (according to J. Nikuradze): 1 - laminar flow; 2 - region of hydraulically smooth turbulence (G. Blasius); 3 - the boundary between the regions of prequadratic and quadratic turbulence; I - transition region between the laminar flow and the region of hydraulically smooth turbulence; II - region of prequadratic turbulence; III region of quadratic turbulence.
In the laminar flow, the fluid moves slowly and calmly. It flows around the roughness of the inner surface of the pipe. The values of local friction drag are small, so they can be neglected.

The friction factor of the laminar flow in the coordinates $\lg (100 \lambda)=f(\lg R e)$ is described by a graph of a straight line (Fig. 2).

In 1838, Poiseuille studied currents in pipes of small diameter (capillaries) with distilled water at low flow discharges (Poiseuille flows). In 1846 he published a law determining the flow discharge of a steady stream of viscous incompressible fluid in a thin cylindrical tube of circular cross-section.

According to this law, the second volume flow discharge is proportional to the pressure drop $\left(p_{1}-p_{2}\right)$ per unit length of pipe $l$ (pressure gradient in the pipe), the fourth degree of the pipe diameter $d$ and inversely proportional to the dynamic viscosity of the flow

$$
Q=K \frac{\left(p_{1}-p_{2}\right) d^{4}}{\mu l}
$$

where $K$ - coefficient of proportionality, $K=\pi / 128-$ theoretically determined by $\mathrm{D}$. Stokes in $1845 ; \mu-$ dynamic viscosity, $\mathrm{Pa} \cdot \mathrm{s}$.

It should be noted that in 1839 this law was first formulated by the G. Hagen. Therefore, equation (4) is called the Hagen - Poiseuille law. The flow discharge in the Hagen-Poiseuille formula was represented by the average velocity and diameter of the pipe, and the resulting expression was compared with the Darcy Weisbach equation. The dependence on determining the friction factor was then obtained.

$$
\lambda=\frac{64}{R e} .
$$

If $R e \leq 2320$, then this formula is correct and describes the line of the laminar flow (Fig. 2). The graph of the given equation in ordinary coordinates has the form of a hyperbola, and in logarithmic coordinates is represented by a straight line.

Turbulent flow moves much faster than laminar flow. This leads to large velocity gradients between the flow layers of the turbulent core. As a result, a vortex flow is formed. All this leads to an increase in hydraulic friction, which in the laminar flow could be neglected.

The laminar layer is in the turbulent flow between the turbulent flow core and the inner surface of the pipe in the region of hydraulically smooth turbulence.

The thickness of the laminar layer of the total flow is greater than the height of the roughness. In this case, the roughness of the inner surface of the pipe has almost no effect on the turbulent core and the head loss does not depend on the roughness.

Many scientists have performed a large amount of experimental and theoretical research to determine the dependences of the influence of major factors on the value of the friction factor.

Currently, there is no general formula for determining the friction factor $\lambda$. 
The topic of this article is the theoretical study of the dependence of the friction factor on the Reynolds number of turbulent flow in hydraulically smooth pipes.

Consider the basic formulas of the friction factor for the region of hydraulically smooth turbulence, which is in the range of values $\left(4000 \leq R e \leq 3 \cdot 10^{6}\right)$.

G. Blasius in 1913 showed that the friction factor is determined by the dependence [1]

$$
\lambda=\frac{0,3164}{R e^{0,25}} .
$$

L. Prandtl in 1932 obtained the dependence on the logarithmic velocity profile and on the basis of the results of experimental studies [2]

$$
\frac{1}{\sqrt{\lambda}}=2 \lg (\operatorname{Re} \sqrt{\lambda})-0,8 .
$$

In 1938, C. Colebrook proposed to determine the friction factor by the formula [3]

$$
\lambda=\frac{1,63639}{\left(\ln 0,142857 \operatorname{Re}()^{2}\right)} .
$$
[4]

P.K. Konakov (1946) presented an empirical formula

$$
\lambda=\frac{1}{(1,8 \lg R e-1,5)^{2}} .
$$

G.K. Filonenko in 1948 recommended the following dependence [6].

$$
\lambda=\left(\frac{0,55}{\lg \frac{R e}{8}}()^{2}\right)
$$

In 1962, M.Y. Ruzin proposed to determine the friction factor by the formula [5]

$$
\lambda=\frac{0,246}{R e^{0,22}} .
$$

F.A. Shevelyov in 1973 determined on the basis of experimental studies the dependence for steel pipes [6]

$$
\lambda=\frac{0,25}{R e^{0,226}} .
$$

Based on mathematical statistics, the Chinese scientist Guo Ningxiang proposed to determine the friction factor by a formula [7]

$$
\frac{1}{\sqrt{\lambda}}=1,689 \lg R e+\frac{0,35}{1-0,084 R e} .
$$

\section{Problem statement}

There are problems in calculating engineering hydraulic problems. When calculating the capacity of pipes, it is not enough to know the main factors: flow viscosity, pipe diameter, the roughness of the inner surface of the pipes. It is necessary to take into account the influence of the hydraulic regime on the value of the friction factor $\lambda$.

Analysis of the presented formulas, in addition to the formula of L. Prandtl (7), shows that in the region of hydraulically smooth turbulence the friction factor $\lambda$ depends only on the Reynolds number, which is in the denominator and has a degree of about 0.25 . The numerator of these formulas contains a coefficient, the physical meaning of which is not explained. The graphs of the above equations, in ordinary coordinates, have the form of a hyperbola, and in logarithmic coordinates are represented by a straight line.

In L. Prandtl's formula, the square root of the friction factor is described in an implicit form and is calculated, like the Reynolds number, under the sign of the logarithm. This form of the formula complicates the determination of the friction factor and does not show the influence of parameters on its value.

The formulas of the friction factor along the length of the pipe, which is included in equation (2) are the subject of our theoretical research.

\section{Research results}

To develop a general method of taking into account the head loss in the flow of real fluid, it is necessary to identify the dependence of friction forces on the main factors. These main factors are fluid density $\rho$, dynamic viscosity $\mu$, hydraulic flow radius $R$, the complex of the linear dimensions that characterize the inner surface of the pipe wall (roughness height, the distance between roughness's and their shape) $\varepsilon_{\mathrm{i}}$ and average flow velocity $\bar{u}$.

Using the method of dimensional analysis, we have established a general view of the dependence of the friction forces on the inner wall of the pipe from these factors [1]

$$
\tau_{0}=k \rho^{z} \mu^{m} R^{p} \varepsilon^{x} \bar{u}^{n} .
$$

In dimensional quantities, equation (14) has the form

$$
\frac{M l}{t^{2}} \frac{1}{l^{2}}=\frac{M^{z}}{l^{3 z}} \frac{M^{m}}{l^{m} t^{m}} l^{p} l_{i}^{x} \frac{l^{n}}{t^{n}}
$$

where $M$ - mass; $l$ - linear dimensions; $t$ - time.

Equated dimensionalities $M, l$, and $t$ in the right and left parts of equation (15) have the form

$$
\begin{gathered}
\text { for the mass: } 1=m+z \\
\text { for linear dimensions: }-1=n+p-m-3 z+x\} \text {. } \\
\text { for time: }-2=-n-m
\end{gathered}
$$

1 and 2 are known parameters and from the system (16), we will define other indicators of degrees

$$
\left.\begin{array}{c}
m=2-n \\
z=n-1 \\
p=n-2-x
\end{array}\right\}
$$

Substituting the found degrees through $n$ and $x$ in equation (14), we obtain the formula of tangential stresses on the inner wall of the pipe.

$$
\tau_{0}=k \rho \frac{\bar{u}^{2}}{R e^{2-n}}\left(\frac{\varepsilon}{R}\right)^{x}
$$

Based on experimental studies, it is known that in a turbulent flow, the value of degree $n=2$. Then the tangential stresses on the pipe wall are 


$$
\tau_{0}=k\left(\frac{\varepsilon}{R}\right)^{x} \rho \bar{u}^{2}=2 k\left(\frac{\varepsilon}{R}\right)^{x} \rho g \frac{\bar{u}^{2}}{2 g},
$$

In order to simplify the calculations, A. Darcy proposed in equation (19) to replace the coefficient of friction $f=2 k\left(\frac{\varepsilon}{R}\right)^{\mathrm{x}}$ by the coefficient of A. Darcy $f=\frac{\lambda}{4}$.

$$
\tau_{0}=\frac{\lambda}{4} \rho g \frac{\bar{u}^{2}}{2 g} .
$$

The right-hand sides of equations (18) and (20) are the same and $\mathrm{x}=1, R=r_{0} / 2$

$$
\lambda=8 k \frac{2 \varepsilon / r_{0}}{R e^{2-n}}
$$

The obtained dependence (20) shows that the dimensionless coefficient in formulas (6) - (12), in addition to L. Prandtl's formula, characterizes the roughness of the inner surface of pipes with degree $n \approx$ 1,75 .

If in formula (20) take $k=8, \mathrm{x}=0$, and $n=1$, then we obtain the dependence (5) to determine the friction factor $\lambda$ of the laminar flow

$$
\lambda=\frac{64}{R e}
$$

Based on experimental studies, it is known that the degree takes its minimum value of $n=1$ for laminar flow and the maximum value of $n=2$ for turbulent flow in the region of quadratic turbulence. It is also known that the inner surface of the pipes affects the amount of friction factor.

We accept two hypotheses:

- degree $n$ in the regions of hydraulically smooth and quadratic turbulence takes intermediate values in the range $1<n<2$;

- to characterize the inner surface of the pipe wall, namely: the average roughness height, the distance between the roughness and their shape is possible only using the relative parameters $2 \varepsilon_{i} / r_{0}$.

Based on the accepted hypotheses, the dependence (20) will have the form

$$
\lambda=64\left(\frac{2 \varepsilon_{1} / r_{0}}{R e^{0,75}}+\frac{2 \varepsilon_{2} / r_{0}}{R e^{0,5}}+\frac{2 \varepsilon_{3} / r_{0}}{R e^{0,25}}+\frac{2 \varepsilon_{4}}{r_{0}}\right) .
$$

Dependence (22) is obtained by the method of dimensional analysis and characterizes the inner surface of the pipes. This formula determines the friction factor depending on the Reynolds number.

The adequacy of equation (22) was verified by the experimental data obtained by J. Nikuradze and A.D. Altshul for the flow in smooth pipes.

J. Nikuradze performed experimental research on three experimental installations. These installations were built in the workshops of the Kaiser Wilhelm Institute.

For small Reynolds numbers from $3 \cdot 10^{3}$ to $60 \cdot 10^{3}$, the pressure in the pipe was created by an overflow tank. The water supply came from the general water supply system. The discharge of water consumption was determined by the volumetric method.

For Reynolds numbers up to $1400 \cdot 10^{3}$, the pressure in the pipe was created using a rotary pump.
To create an even larger Reynolds number (up to $2500 \cdot 10^{3}$ ), the pressure in the pipe was increased by compressed air in a closed pressure tank.

In the last two installations, the Reynolds number was further increased by increasing the water temperature.

When conducting experiments on such installations, J. Nikuradze expanded the range of research to large values of Reynolds numbers. The maximum possible value of the Reynolds number in the study was $R e=3300 \cdot 10^{3}$.

The results of J. Nikuradze's experiments in the region of hydraulically smooth turbulence [2] are graphs in coordinates $\lg (100 \lambda)=f(\lg \operatorname{Re}())$ in Figure 3 .

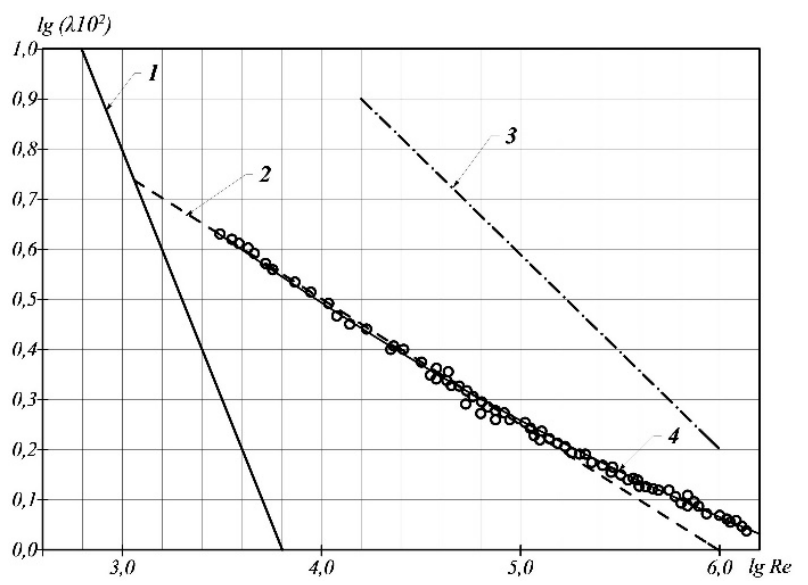

Fig. 3. Graphs of the dependence of the friction factor on the Reynolds number (according to J. Nikuradze): 1 - laminar flow; 2 - the region of hydraulically smooth turbulence (G. Blasius); 3 - the boundary between the regions of prequadratic and quadratic turbulence; 4 - according to the author's formula; $\mathrm{O}$ experimental results of J. Nikuradze.

The figure 3 shows that the friction factor in coordinates $\lg (100 \lambda)=f(\lg R e)$ depending on the main factors and the hydraulic regime within the regions takes different values.

Table 1 shows the diameters of the pipes and the Reynolds number, in the range of which hydraulic studies were performed by J. Nikuradze. The table also presents the dispersion of adequacy, which characterize the discrepancy of the experimental data relative to the theoretical formulas (6) - (12), (22).

The least squares method gave the values of the elements of the complex of relative parameters: $2 \varepsilon_{1} / r_{0}=$ $0, \quad 2 \varepsilon_{2} / r_{0}=0,01034, \quad 2 \varepsilon_{3} / r_{0}=0,003124$, $2 \varepsilon_{4} / r_{0}=0,0000726$. Then equation (22) has the form

$$
\lambda=64\left(\frac{0,01034}{R e^{0,5}}+\frac{0,03124}{R e^{0,25}}+0,0000726\right) .
$$

The table 1 shows that the dispersion of adequacy of experimental data with the formula of G. Blasius increases with increasing Reynolds number. The dispersion of adequacy with the formulas of L. Prandtl, C. Colebrook, P.K. Konakov, G.K. Filonenko, F.A. Shevelyov and with the formula of the authors of this article decreases with increasing Reynolds number. The dispersion of adequacy of experimental data with the M.Y. Ruzin formula with increasing Reynolds numbers first increases and then decreases. 
Table 1. The dispersion of adequacy of experimental data of hydraulic studies of smooth pipes obtained by J. Nikuradze, relative to the recommended formulas.

\begin{tabular}{|c|c|c|c|c|c|c|c|}
\hline \multirow{4}{*}{ Author } & \multirow{4}{*}{$\mid \begin{array}{c}\text { Formula } \\
\text { number }\end{array}$} & \multicolumn{6}{|c|}{$\begin{array}{c}\text { Dispersion of adequacy } D^{2} \times 10^{6} \text { for } \\
\text { pipe diameters, mm and Reynolds } \\
\text { numbers } \frac{\operatorname{Re} \operatorname{mim} \times 10^{-3}}{\operatorname{Re} \max \times 10^{-3}}\end{array}$} \\
\hline & & 10 & 20 & 30 & 50 & 100 & $\begin{array}{l}10- \\
100\end{array}$ \\
\hline & & \begin{tabular}{|l|}
3,07 \\
\end{tabular} & 17 & 106 & 37 & 318,9 & 3,07 \\
\hline & & 23 & $\overline{182}$ & $\overline{288}$ & $\overline{670}$ & $\overline{1364}$ & $\overline{1364}$ \\
\hline G. Blasius & (6) & 0,167 & 0,251 & 0,293 & 0,433 & 2,835 & 1,348 \\
\hline L. Prandtl & (7) & 0,336 & 0,210 & 0,050 & 0,082 & 0,023 & 0,112 \\
\hline C. Colebrook & (8) & 0,401 & 0,203 & 0,046 & 0,103 & 0,026 & 0,123 \\
\hline P.K. Konakov & (9) & 0,449 & 0,239 & 0,059 & 0,152 & 0,036 & 0,150 \\
\hline $\begin{array}{l}\text { G.K. Filonen- } \\
\text { ko }\end{array}$ & (10) & 6 & 0,202 & 0,048 & 0,069 & 0,023 & 0,196 \\
\hline M.Y. Ruzin & (11) & 0,877 & 3,310 & 1,732 & 2,210 & 0,244 & 1,417 \\
\hline $\begin{array}{c}\text { F.A. Shevely- } \\
\text { ov }\end{array}$ & (12) & 1,572 & 0,694 & 0,160 & 0,342 & 0,573 & 0,645 \\
\hline $\begin{array}{c}\text { Guo } \\
\text { Ningxiang }\end{array}$ & (13) & 0,358 & 0,134 & 0,053 & 0,090 & 0,021 & 0,116 \\
\hline $\begin{array}{l}\text { Authors of } \\
\text { the article }\end{array}$ & (23) & 0,150 & 0,194 & 0,041 & 0,069 & 0,024 & 0,083 \\
\hline
\end{tabular}

If we analyze the dispersion of the adequacy of experimental formulas in Table 1, then at Reynolds numbers from 3070 to 1364000 - minimal errors give the formula of the authors of the article and the formula of L. Prandtl, C. Colebrook and G.K. Filonenko. The biggest errors are given by the formulas of G. Blazius and M.Y. Ruzin.

A.D. Altshul performed experimental research on hydraulic and aerodynamic installations [8].

Aluminum pipes had a smooth inner surface and were coated with oil. Before the experiments, they were washed with solvent and thoroughly wiped with rags. Pipes with a diameter of $350 \mathrm{~mm}$ had a small roughness, formed by insufficiently clean broaching (during their manufacture) and coating of alumina oxide.

The tested sections of pipes were always made of solid pipe (there were no joints on the section). The inlet and outlet sections were made of the same aluminum pipes as the measuring section.

The study of an aluminum pipe with a diameter of 26 $\mathrm{mm}$ was performed on a hydraulic installation, and with a diameter of 109.8 and $350 \mathrm{~mm}$ - on an aerodynamic installation.

The results of experimental studies of A.D. Altshul in the region of hydraulically smooth turbulence [8] are shown in Figure 4 in coordinates $\lg (100 \lambda)=f(\lg \operatorname{Re}())$.

Table 2 shows the diameters of the pipes and the Reynolds number, in the range of which hydraulic studies were performed by A.D. Altshul. The table also presents the dispersion of adequacy, which characterizes the discrepancy of the experimental data relative to the theoretical formulas (6) - (12), (22).

If we analyze the dispersion of the adequacy of experimental data (table 2) relative to the formulas for Reynolds numbers from 2844 to 338844 , the minimal errors give the formulas of the authors of this article, L. Prandtl, C. Colebrook, G.K. Filonenko, and F.A. Shevelev.

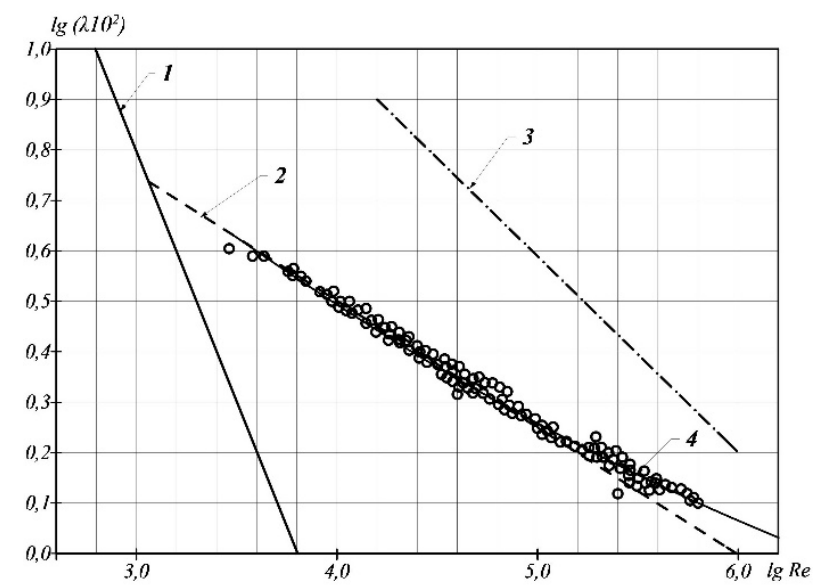

Fig. 4. Graphs of the dependence of the friction factor on the Reynolds number: 1 - laminar flow; 2 - the region of hydraulically smooth turbulence (G. Blasius); 3 - the boundary between the regions of prequadratic and quadratic turbulence; 4 - according to the author's formula; $\mathrm{O}$ - experimental results of A.D. Altshul.

Table 2. The dispersion of adequacy of experimental data of hydraulic studies of smooth pipes obtained by A.D. Altshul, relative to the recommended formulas

\begin{tabular}{|c|c|c|c|c|c|c|c|}
\hline \multirow{4}{*}{ Author } & \multirow{4}{*}{$\begin{array}{c}\text { For- } \\
\text { mula } \\
\text { num } \\
\text { ber }\end{array}$} & \multicolumn{6}{|c|}{$\begin{array}{l}\text { Dispersion of adequacy } D^{2} \times 10^{6} \text { for pipe } \\
\text { diameters, mm and Reynolds numbers } \\
\qquad \frac{R e \operatorname{mim} \times 10^{-3}}{\operatorname{Re} \max \times 10^{-3}}\end{array}$} \\
\hline & & 18,45 & 26 & 26 & 109,8 & 350 & $\begin{array}{c}18,45- \\
350\end{array}$ \\
\hline & & 2884 & 19486 & 9550 & 141254 & 251189 & 2884 \\
\hline & & 38905 & $\overline{33113}$ & 25119 & 285102 & 338844 & 338844 \\
\hline $\begin{array}{c}\text { G. Bla- } \\
\text { sius } \\
\end{array}$ & (6) & 0,509 & 0,286 & 1,128 & 0,324 & 1,239 & 0,690 \\
\hline $\begin{array}{c}\text { L. Pran } \\
\text { dtl }\end{array}$ & (7) & 1,420 & 0,249 & 2,207 & 0,078 & 0,192 & 0,571 \\
\hline $\begin{array}{l}\text { C. Co- } \\
\text { lebrook }\end{array}$ & (8) & 1,879 & 0,265 & 2,373 & 0,050 & 0,204 & 0,651 \\
\hline $\begin{array}{c}\text { P.K. } \\
\text { Kona- } \\
\text { kov }\end{array}$ & (9) & 1,995 & 0,371 & 2,794 & 0,044 & 0,226 & 0,763 \\
\hline $\begin{array}{c}\text { G.K. } \\
\text { Filo- } \\
\text { nenko }\end{array}$ & (10) & 1,279 & 0,187 & 1,602 & 0,083 & 0,192 & 0,559 \\
\hline $\begin{array}{l}\text { M.Y. } \\
\text { Ruzin }\end{array}$ & (11) & 0,866 & 2,675 & 0,353 & 2,176 & 0,874 & 1,595 \\
\hline $\begin{array}{c}\text { F.A. } \\
\text { Sheve- } \\
\text { lyov }\end{array}$ & (12) & 0,292 & 0,516 & 0,588 & 0,341 & 0,213 & 0,389 \\
\hline $\begin{array}{c}\text { Guo } \\
\text { Ning- } \\
\text { xiang } \\
\end{array}$ & (13) & 1,609 & 0,264 & 2,234 & 0,078 & 0,192 & 0,612 \\
\hline $\begin{array}{c}\text { Aut- } \\
\text { hors of } \\
\text { the ar- } \\
\text { ticle }\end{array}$ & (23) & 1,167 & 0,199 & 1,713 & 0,066 & 0,199 & 0,477 \\
\hline
\end{tabular}

The biggest errors are given by the formula M.Y. Ruzin.

Table 2 shows that the dispersion of the adequacy of experimental data for the formula of G. Blasius with increasing Reynolds number increases. For the formulas of L. Prandtl, C. Colebrook, P.K. Konakov, G.K. Filonenko, F.A. Shevelyov, and the formulas of the 
authors of the article with increasing Reynolds number, the dispersion of adequacy decreases.

The good results of L. Prandtl's formula can be explained by the fact that this dependence is obtained theoretically from the logarithmic velocity profile.

All other formulas are obtained by statistical methods, so the determined results differ significantly from the experimental data.

The given experimental researches of J. Nikuradze and A.D. Altshul were performed for pipes of different material and diameter, on hydraulic and aerodynamic installations with the use of different measuring equipment.

The experimental data of A.D. Altshul (Figs. 3, 4) have a greater dispersion of adequacy. This can be explained by the fact that the experiments were performed using water and air. But all studies have a wide range of Reynolds numbers.

It should be noted that the determination of pipe diameters, pipe lengths between piezometric pressure sampling points, pressure drops, and water discharges were determined by calibrated equipment.

The accuracy of all experiments is high because there are no systematic errors. All experimental data are grouped only near one theoretical line by dependence (23). There are random errors, and they are greater in the experiments of A.D. Altshul.

We can conclude that the described experiments of J. Nikuradze and A.D. Altshul are sufficient for theoretical research.

The formula of the authors of the article gives good results because it is obtained on the basis of the method of dimensional analysis, characterizes the roughness of the inner surface of the pipes. This formula also takes into account the influence of Reynolds numbers on the friction factor.

\section{Conclusions and prospects for future research}

The article presents the results of theoretical studies of the flow friction factor in hydraulically smooth pipes. The dependence for the calculation of the friction factor based on the method of dimensional analysis is obtained. The adequacy of the author's equation and experimental data of J. Nikuradze and A.D. Altshul in the study of water flow in smooth pipes is determined.

It is shown that in almost all cases, formula (23) gives the best results. This formula is obtained on the basis of the method of dimensional analysis, which characterizes the roughness of the inner surface of the pipes. It also takes into account the influence of Reynolds numbers on the friction factor.

Therefore, formula (23) can be recommended to determine the friction factor of turbulent flow in the region of hydraulically smooth turbulence.

Future articles will present the results of theoretical studies of the friction factor for the regions of prequadratic and quadratic turbulence in steel and aluminum pipes.

\section{References}

1. A.I. Bogomolov, K.A. Mikhailov, Hydraulics (Stroyizdat, Moscow, 1942)

2. J. Nikuradse, Gesetzmassigkeiten der turbulenten Strömung in glatten Rohren, (Forsch. Arb. Ing. Wes., 1932)

3. J. Nikuradse, Strömungsgesetze in rauchen Rohren, (Forsch. Ver. Dtsch. Ing., 1933)

4. Colebrook C.F., White C.M., Experiments with Fluid Friction in Roughened Pipes, (Proceedings of the Royal Society of London. Series A, Mathematical and Physical Sciences, 1937)

5. M.Y. Ruzin, Hydraulic calculation of plastic pipelines (Water supply and sanitary engineering, 1962)

6. F.A. Shevelev, Investigation of the basic hydraulic laws of turbulent motion in pipes, (Gosstroyizdat, Moscow, 1953)

7. A.D. Altshul, V.I. Kalitsun, Hydraulic resistances of pipelines, (Gosstroyizdat, Moscow, 1964)

8. Guo Nianxiang, Liao Chunfa, Wan Linsheng, Liu Jianhua, $\mathrm{Xu}$ Zhifeng, Approximate solution of Nikuradse-Karman equation, (Journal of Southern Institute of Metallurgy, 2002, 01) 